\title{
Acoustic Nature of the Boson Peak in Vitreous Silica
}

\author{
C. Masciovecchio ${ }^{1}$, V. Mazzacurati ${ }^{2}$, G. Monaco $^{2}$, G. Ruocco ${ }^{2}$, T. Scopigno ${ }^{2}$, F. Sette ${ }^{1}$, P. Benassi $^{2}$, A. Cunsolo ${ }^{1}$,

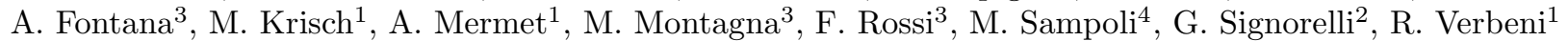 \\ 1 European Synchrotron Radiation Facility, BP 220, F-38043, Grenoble, Cedex, France. \\ 2 Dipartimento di Fisica and INFM, Università di L'Aquila, I-67100, L'Aquila, Italy. \\ 3 Dipartimento di Fisica and INFM, Universitá di Trento, I-38100, Trento, Italy. \\ 4 Dipartimento di Energetica and INFM, Universitá di Firenze, I-50139, Firenze, Italy.
}

(May 24, 2022)

\begin{abstract}
New temperature dependent inelastic x-ray (IXS) and Raman (RS) scattering data are compared to each other and with existing inelastic neutron scattering data in vitreous silica $\left(v-\mathrm{SiO}_{2}\right)$, in the $300 \div 1775 \mathrm{~K}$ region. The IXS data show collective propagating excitations up to $Q=3.5 \mathrm{~nm}^{-1}$. The temperature behaviour of the excitations at $Q=1.6 \mathrm{~nm}^{-1}$ matches that of the boson peak found in INS and RS. This supports the acoustic origin of the excess of vibrational states giving rise to the boson peak in this glass.
\end{abstract}

PACS numbers : 63.10.+a, 63.50+x, 78.70.Ck

A peculiar characteristic of disordered solids, not present in their crystalline counterpart (Phillips, 1981), is an excess of low frequency states with respect to the predictions of the Debye theory. Inelastic neutron scattering (INS) studies on the density of states, $g(\omega)$, reveal, in fact, a broad peak (the boson peak) (BP) in the quantity $g(\omega) / \omega^{2}$ (Buchenau et al. 1986, Buchenau et al. 1988, Sokolov et al. 1995) at energies $2 \div 10 \mathrm{meV}(15 \div 80$ $\mathrm{cm}^{-1}$ ), where the Debye theory would predict a constant. This excess is responsible for the presence, in any glass studied so far, of a bump in the temperature dependence of $C_{p} / T^{3}$ (Sokolov et al. 1993, Brodin et al. 1994).

The broad boson peak feature is also observed in Raman scattering (RS) spectra. This can be understood considering that the first order Raman scattering intensity, $I(\omega)$, in the harmonic approximation, is connected to $g(\omega)$ (Galeneer and Sen 1978): $I(\omega)=$ $C(\omega) g(\omega)[n(\omega, T)+1] / \omega$. Here $n(\omega, T)$ is the Bose population factor and $C(\omega)$ is the photon-excitation coupling function, assumed to have a smooth behaviour in the considered energy region (Fontana, Rocca and M. P. Fontana 1987, Fontana et al. 1997).

The nature of this excess of states is still subject to speculation and, at present, there are two different prevailing hypotheses (See Philosophical Magazine, special issue: $V$ international workshop on disordered systems, Andalo, 1994). In the first one, the excess of states is explained by the localization of the high energy vibrational modes induced by the static disorder in the glass. In the second case, collective propagating modes are thought to persist at high frequency, and the $\mathrm{BP}$ reflects their density of states. In the first view the BP energy corresponds to the energy of the high $Q$ excitations, while in the second one the excitations are expected to propagate at energies above the BP energy.

Information on the character of the excitations contributing to the boson peak can be obtained from measurements of the dynamic structure factor $S(Q, \omega)$. Using inelastic x-ray scattering (IXS) (Sette et al. 1995), it has been possible to measure $S(Q, \omega)$ of different glasses in the momentum-energy region of the $\mathrm{BP}$, and to support the hypothesis of the acoustic-like character of the collective modes and therefore of the peak itself (Masciovecchio et al. 1996a, Masciovecchio et al. subm.). In the specific case of $v$-silica, however, the IXS data have been alternatively interpreted either with the phonon-localization model (Foret et al. 1997), or as propagating excitations (Benassi et al 1997, Masciovecchio et al. 1997).

A better insight on the relation between the excitations seen in $S(Q, \omega)$ and the $\mathrm{BP}$ could be gained by comparing the temperature dependence of their energies. The basic idea is to utilise the observation that in vitreous silica, in contrast to the majority of glasses, the low frequency sound velocity, measured by Brillouin Light Scattering (BLS), (Vacher and Pelous 1976) and the boson peak energy (Wischnewski et al. 1998) shift towards higher values with increasing temperatures, and, more importantly, the rates of change reported in the two cases are different. It is not the aim of this work to account for these peculiarities, likely to be due to the specific shape of the interatomic potential in silica. Rather, one can utilize the different temperature dependence of excitations with different $Q$, as seen by IXS, INS, RS and BLS, to establish a link between specific vibrational states and the features of the density of states. In particular, one can assess whether the excitations observed by IXS at energies comparable to the BP energy have either a temperature behaviour similar to that of the $\mathrm{BP}$, or to that of the low $Q$ excitations measured by BLS, or even another dependence.

In this Letter we report temperature dependent IXS and RS data of vitreous silica. In the IXS spectra, propagating collective excitations are found at $T \approx 1400 \mathrm{~K}$, thus confirming that the high frequency longitudinal dynamics has an acoustic-like nature in the whole temperature region below melting, and up to a momentum transfer $Q=3.5 \mathrm{~nm}^{-1}$, corresponding to energies more than twice the BP energy $\left(E_{B P} \approx 4 \mathrm{meV}\right.$ at room tempera- 
ture). More importantly, we find the same temperature shift for the BP energy and for the energy, $\Omega(Q)$, of the modes at $Q^{*}=1.6 \mathrm{~nm}^{-1}\left(\Omega\left(Q^{*}\right) \approx E_{B P}\right)$. This supports the argument that the acoustic modes, probed by the IXS, give a significant contribution to the BP.

The IXS experiment was carried out at the very high energy resolution inelastic x-ray scattering beamline (BL21/ID16) at the European Synchrotron Radiation Facility. The total resolution function, measured using a Plexiglas scatterer at the maximum of its static structure factor $\left(Q=10 \mathrm{~nm}^{-1}\right)$, has a full width at half maximum (fwhm) of $1.5 \pm 0.1 \mathrm{meV}$. The $Q$ values were selected between 0.75 and $4 \mathrm{~nm}^{-1}$, and the $Q$ resolution was set to $0.2 \mathrm{~nm}^{-1} \mathrm{fwhm}$. Energy scans were performed by varying the relative temperature between the monochromator and the analyser crystals by $\pm 0.45 \mathrm{~K}$ with a step of 0.0075 K. Each scan took about $200 \mathrm{~min}$, and each $Q$-value was obtained by averaging five scans (the total integration time was $500 \mathrm{~s}$ per point). The data were normalized to the intensity of the incident beam. Further details on the IXS beamline are reported elsewhere (Masciovecchio et al. 1996a, b, Verbeni et al. 1996). The Raman scattering measurements were performed using a standard Raman laser system. Depolarized spectra were collected in the $-300 \div 2000 \mathrm{~cm}^{-1}$ frequency range with $1 \mathrm{~cm}^{-1}$ frequency resolution. The $\mathrm{SiO}_{2}$ suprasil sample, purchased from Goodfellow, was the same $2 \mathrm{~mm}$ diameter rod used in (Benassi et al 1997, Masciovecchio et al. 1997).

From the linear dispersion curve determined by a first set of IXS spectra taken at $T=1375 \mathrm{~K}$, it was possible to establish that the excitations have the same propagating nature as that previously observed in the $300 \div 1000$ $\mathrm{K}$ region. In Fig. 1, we report as an example the IXS spectrum taken at $Q=1 \mathrm{~nm}^{-1}$, together with the fitting function. As in previous works, the IXS spectra were fitted by the experimentally determined resolution function convoluted with a $\delta$-function for the elastic peak and a Damped Harmonic Oscillator (DHO) model (Fak and Dorner, 1992) for the inelastic signal. The excitation energies are reported in the inset. The data show an acoustic like behaviour, linearly extrapolating at small $Q$ with a slope $v=6800 \pm 200 \mathrm{~m} / \mathrm{s}$. This value, larger than the sound velocity measured by Brillouin light scattering (Vacher et al. 1976) implies a deformation of the acoustic branch not recognized before, which will provide an interesting subject to further investigations. The dispersion relation covers the whole $\mathrm{BP}$ energy region $(\approx 5 \div 7$ $\mathrm{meV}$ ), and reaches energies as high as $15 \div 20 \mathrm{meV}$. These observations, as in previous lower temperature measurements, show that, even at temperatures approaching $T_{g}$, it exists an acoustic-like propagating mode up to energies that lie well above $E_{B P}$.

A second set of IXS measurements was performed as a function of temperature at the fixed value $Q^{*}=1.6$ $\mathrm{nm}^{-1}$, which was chosen since $\Omega\left(Q^{*}\right)$ is in the range of $E_{B P}$ at room temperature. The IXS data and the corre- sponding fits are shown in Fig. 2. It is possible to observe directly from the row data that the intensity and energy of the inelastic excitation increase with $T$. This is emphasized in the inset of Fig. 2, where the $T$-dependence of the inelastic-to-elastic intensity ratio, $R(T)$, and the excitation energy, $\Omega\left(Q^{*}, T\right)$, are reported.

To compare the $T$-dependence of $\Omega\left(Q^{*}, T\right)$ with that of other spectroscopic features, we define the scaling factor $a_{I X S}(T)$ as $\Omega\left(Q^{*}, T\right) / \Omega\left(Q^{*}, T=0 K\right)$. Here $\Omega\left(Q^{*}, T=0 K\right)$ is the $T=0$ extrapolation of the measured $\Omega\left(Q^{*}, T\right)$. The values of $a_{I X S}(T)$ are reported in Fig. 3, together with the scaling factor for the low $Q$ $\left(Q \approx 0.036 \mathrm{~nm}^{-1}\right)$ excitation energy determined by Brillouin light scattering (Scopigno 1997), $a(T)_{B L S}$. The difference in the $T$-dependence of the low- and high- $Q$ excitations indicates again that the acoustic modes dispersion relation is deformed from a simple linear law. This behaviour was hidden in the error bars in previous IXS and BLS studies (Benassi et al 1997, Masciovecchio et al. 1997). and is evidenced here thanks to increased sensitivity and temperature range. It is not the aim of the present work to discuss the origin of the observed deformation of the dispersion relation 7 .

This non trivial $T$-dependence can help us to assess the origin of the BP by the study of the temperature dependence of its energy. In Fig. 3 we report, in fact, the scaling factor $a_{I N S}(T)$ of the BP energy maximum, as determined from existing INS measurements (Wischnewski et al. 1998). The striking similarity between $a_{I N S}(T)$ and $a_{I X S}(T)$ provides a first strong indication that the states probed by IXS contribute to the BP. This similarity is further confirmed by the temperature dependence of the BP energy as measured by RS in the $300 \div 1100$ $\mathrm{K}$ region. In Fig. 4 we report $I(\omega) \omega / C(\omega) /[n(\omega, T)+1]$, which corresponds directly to $g(\omega) / \omega^{2}$. We used the coupling coefficient $C(\omega)$ derived from the ratio of the INS (Wischnewski et al. 1998) and RS (this work) data measured in $v-\mathrm{SiO}_{2}$ at room temperature: this $C(\omega)$ is reported in the inset a) of Fig. 4, and it was used to scale all the RS spectra at different temperatures I. The energy of the maximum of the spectra of Fig. 4, derived by a local quadratic fit, is reported in inset b) and the scaling factors $a_{R S}(T)$ are reported in Fig. 3. The two data sets (INS and RS) are equivalent within the error bar.

In presence of the deformation of the dispersion re-

\footnotetext{
${ }^{*}$ This effect could, for example, be ascribed to the presence of a positive fourth order anharmonicity in the next-nearest neighbours interaction potentials (Scopigno 1997) or to a relaxation process active in the glass.

${ }^{\dagger}$ It has been recently shown that the coupling function is not dependent on temperature in the energy region spanned by the BP, (A. Fontana et al. subm.).
} 
lation, it is striking to observe the identical temperature behaviour for two spectroscopic features that in principle may share only a similar energy: the $\mathrm{BP}$ maximum and the acoustic excitations at $Q^{*}=1.6 \mathrm{~nm}^{-1}$. This demonstrates their common origin. Consequently, the high frequency acoustic excitations, in spite of their propagating nature, must have a density of states exceeding the Debye prediction.

A model, that is consistent with a picture of linearly dispersing propagating excitations with an excess of states, must take into account the effect of the topological disorder on the eigenvectors of the vibrational modes. It has been shown by molecular dynamics simulation that, in a glass up to the BP energy region, the eigenvector of a given mode $j$ can be thought as the sum of two very different components. i) A plane wave like part, with an average momentum $Q_{j}$, related to the eigenvalue by the linear dispersion $\omega_{j} \approx v Q_{j}$. The $Q$ distribution around $Q_{j}$ gets broader increasing the mode energy. ii) A random uncorrelated component whose spatial Fourier transform extends from $Q_{j}$ up to $Q$ values much higher than the first sharp diffraction peak position with a flat spectral distribution (Mazzacurati, Ruocco and Sampoli 1996). The first component accounts for the peaks in the dynamic structure factor and for the existence of a dispersion relation. The finite width of the inelastic peaks in the $S(Q, \omega)$, as that in Fig. 1, is due to the finite projection of eigenmodes of different energy at the considered $Q$. The presence of the second component allows to satisfy the eigevectors orthogonality conditions without the constrain on mode counting holding for plane waves, i. e. without imposing the $Q^{2}$ dependence of the modes density at a given frequency. Specifically, with respect to the Debye behaviour, this can give rise to an accumulation of states at low energy.

In conclusion, a study on the temperature dependence of the dynamics in vitreous silica has allowed to identify equivalent temperature behaviours for the maximum of the BP and for the high frequency continuation of the sound branch at the same energy. This provides arguments in favour of the acoustic-like origin for the excess in the density of states in vitreous silica. Reminding that the BP is observed basically in any disordered material, and that IXS has shown the presence of a propagating collective dynamics in all the glasses and liquids studied so far, it is natural to speculate on the generality of the conclusion reached here on the nature of the BP.

We acknowledge A. P. Sokolov and G. Viliani for useful discussions.

\section{REFERENCES}

For a review see Amorphous Solids : Low-Temperature properties, edited by W.A. Phillips, (Springer, Berlin, 1981).

BENASSI P., KRISH M., MASCIOVECCHIO C., MONACO G., MAZZACURATI V., RUOCCO G.,
SETTE F. and VERBENI R., 1997, Phys. Rev. Lett. $\mathbf{7 7}, 3835$.

BRODIN A., FONTANA A., BORJESSON L., CARINI G. and TORELL L. M., 1994, Phys. Rev. Lett. 73, 2067.

BUCHENAU U., PRAGER M., NUKER N., DIANOUX A. J., AHMAD N. and PHILLIPS W. A., 1986, Phys. Rev. 34, 5665.

BUCHENAU U., ZHOU H. M., NUKER N., GILROY K. S., and PHILLIPS W. A., 1988, Phys. Rev. Lett. 60, 1318.

FAK B. and DORNER B., 1992, Institute Laue Langevin (Grenoble, France), technical report No. 92FA008S.

FONTANA A., ROCCA F. and FONTANA M. P., 1987, Phys. Rev. Letters 58, 503.

FONTANA A., ROSSI F., CARINI G., D 'ANGELO G., TRIPODO G. and BARTOLOTTA A., 1997, Phys. Rev. Letters 58, 1078.

FONTANA A., DELL 'ANNA R., MONTAGNA M., ROSSI F., VILIANI G., RUOCCO G., SAMPOLI M., BUCHENAU U. and WISCHNEWSKI A., submitted to Europhys. letters

FORET M., COURTENS E., VACHER R. and SUCK J. B., 1997, Phys. Rev. Lett. 77, 3831.

GALENEER F. L. and SEN P. N., 1978, Phys. Rev. $B \mathbf{1 7}, 1928$.

MASCIOVECCHIO C., RUOCCO G., SETTE F., KRISH M., VERBENI R., BERGMAN U. and SOLTWISCH M., 1996, Phys. Rev. Lett. 76, 3356.

MASCIOVECCHIO C., BERGMAN U., KRISH M., RUOCCO G., SETTE F. and VERBENI R., 1996, Nucl. Inst. and Meth. B-111, 181 and B-117, 339

MASCIOVECCHIO C., RUOCCO G., SETTE F., BENASSI P., CUNSOLO A, KRISH M., MAZZACURATI V., MERMET A., MONACO G. and VERBENI R., 1997, Phys. Rev. B55, 8049.

MASCIOVECCHIO C., MONACO G., RUOCCO G., SETTE F., CUNSOLO A., KRISH M., MERMET A., SOLTWISCH M. and VERBENI R., submitted to Phys. Rev. Lett.

MAZZACURATI V., RUOCCO G. and SAMPOLI M., 1996, Europhys. Lett. 34, 681.

SCOPIGNO T., 1997, Thesis, University of L'Aquila. Unpublished.

SETTE F., RUOCCO G., KRISH M., BERGMAN U., MASCIOVECCHIO C., MAZZACURATI V., SIGNORELLI G. and VERBENI R., 1995, Phys. Rev. Lett. $\mathbf{7 5}, 850$.

SOKOLOV A. P., BUCHENAU U., STEFFEN W., FRICK B. and WISCHNEWSKI A., 1995, Phys. Rev. B 52, R9815.

SOKOLOV A. P., KISLIUK A., QUITMANN. D. and DUVAL E., 1993, Phys. Rev. B 48, 7692.

See for example Philosophical Magazine, 1995, B 71, 471-811. Special issue: $V$ international workshop on dis- 
ordered systems, Andalo, 1994, A. Fontana and G. Viliani Editors.

VACHER R. and PELOUS J., 1976, Phys. Rev. B14, 823.

VERBENI R., SETTE F., KRISH M., BERGMAN U., GORGES B., HALCOUSSIS C., MARTEL K., MASCIOVECCHIO C., RIBOIS J.F., RUOCCO G. and SINN H., 1996, J. of Synchrotron Radiation 3, 62.

WISCHNEWSKI A., BUCHENAU U., DIANOUX A. J., KAMITAKARHA W. A. and ZARESTKY J. L., 1998, Phil. mag. B77, 579.

\section{FIGURE CAPTIONS}

FIG. 1 - Inelastic x-ray scattering spectrum of $v-\mathrm{SiO}_{2}$ at $\mathrm{T}=1375$ $\mathrm{K}$ and $Q=1 \mathrm{~nm}^{-1}$ (o). The full line is the best fit to the data as discussed in the text. The dashed and dotted lines represent the elastic and inelastic contributions to the fit. The inset reports the energy of the excitations as derived from the fits. The dashed line is the linear extrapolation to small $Q$, and its slope corresponds to $6800 \mathrm{~m} / \mathrm{s}$.

FIG. 2 - X-ray spectra of $v-\mathrm{SiO}_{2}$ at $Q=1.6 \mathrm{~nm}^{-1}$ taken at different temperatures. The data are shown together with their best fits (full line) and the individual contributions to the fitting function: elastic peak (dotted line), and inelastic components (dashed line). In the inset are reported: 1) (•) the inelastic-to-elastic intensity ratio, $R(T)$; it follows the expected linear behaviour; and 2) (o) the excitation energy, $\Omega(T)$, derived from the DHO model. The reported full lines are their linear fits.

FIG. 3 - Temperature dependence of the scaling factor $a(T)$, normalized to $a(0)=1$, for the different spectra. Open symbols refer to incoherent measurements of the BP: INS (open circles) and Raman scattering (open squares). Full symbols are the scaling factors for the excitations energies determined from the dynamics structure factor $S(Q, \omega)$ measured at $Q=1.6 \mathrm{~nm}^{-1}$ by IXS (full circles) and at $Q=0.036 \mathrm{~nm}^{-1}$ by BLS (full squares). The dashed lines are linear guides to the eye to emphasise differences and similarities among the temperature behaviours of the four data sets.

FIG. 4 - Examples of RS spectra taken, from to top to bottom, at $333,523,823$, and $1073 \mathrm{~K}$. The reported data correspond to the reduced Raman intensities divided by the $C(\omega)$ (Fontana et al. submitted) and shown in inset a). These spectra correspond to the $g(\omega) / \omega^{2}$. In inset b), the energy position of the maximum intensity at each temperature $(\bullet)$ is reported togheter with its linear fit in the low temperature region (dashed line). 


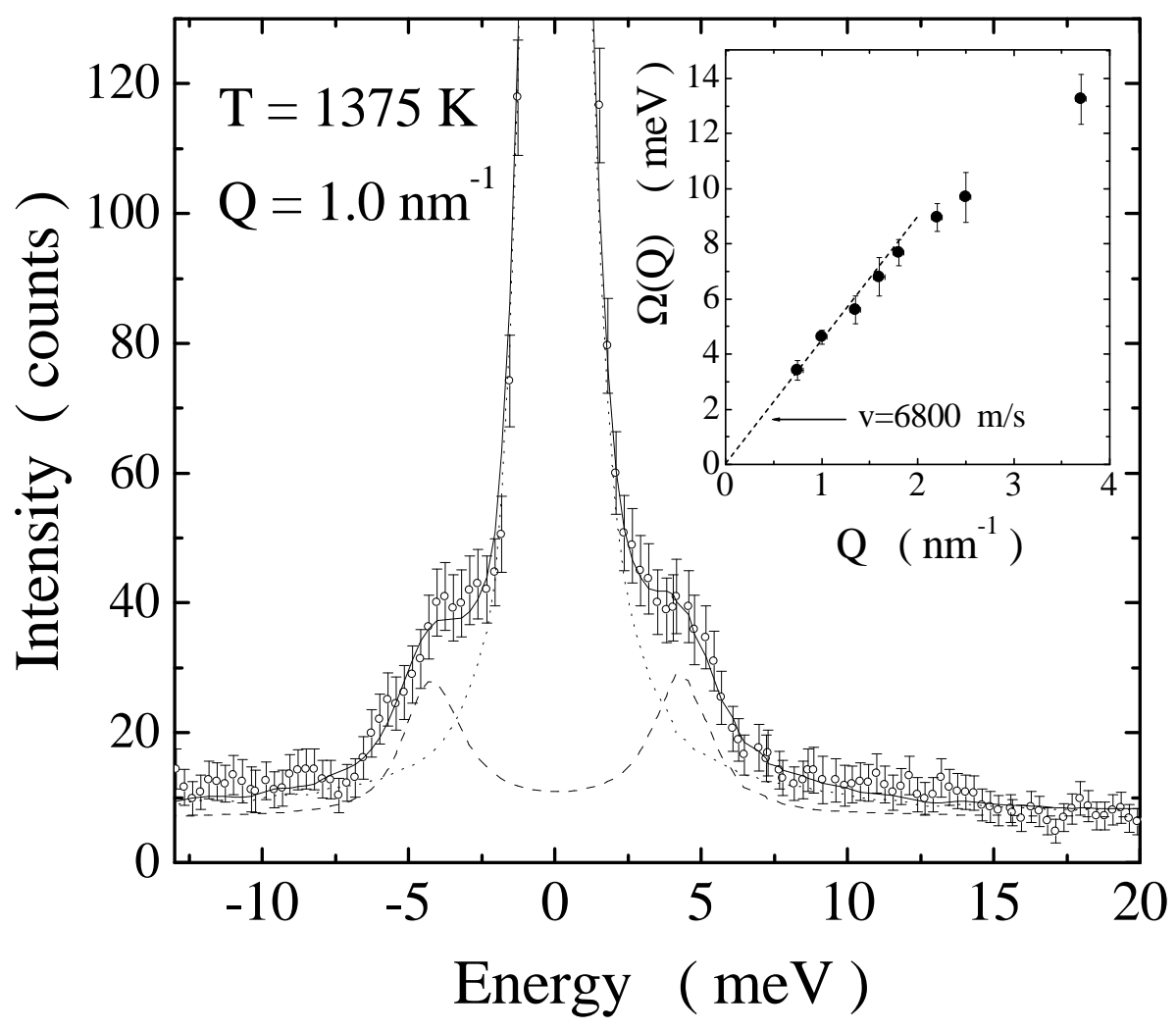

Figure 1: "Acoustic Nature of the Boson Peak...", by C. Masciovecchio et al. 


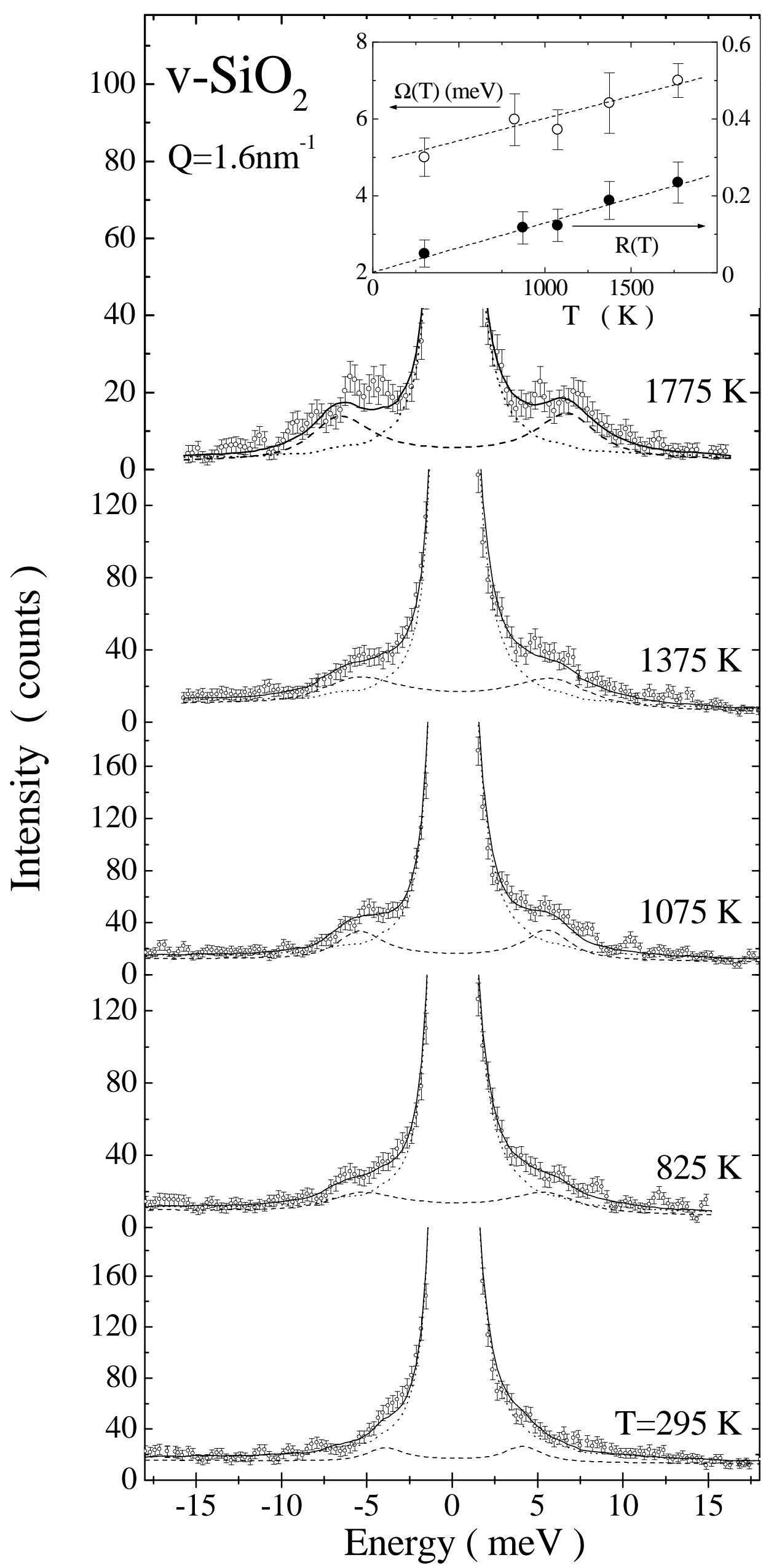

Figure 2: "Acoustic Nature of the Boson Peak...", by C. Masciovecchio et al. 


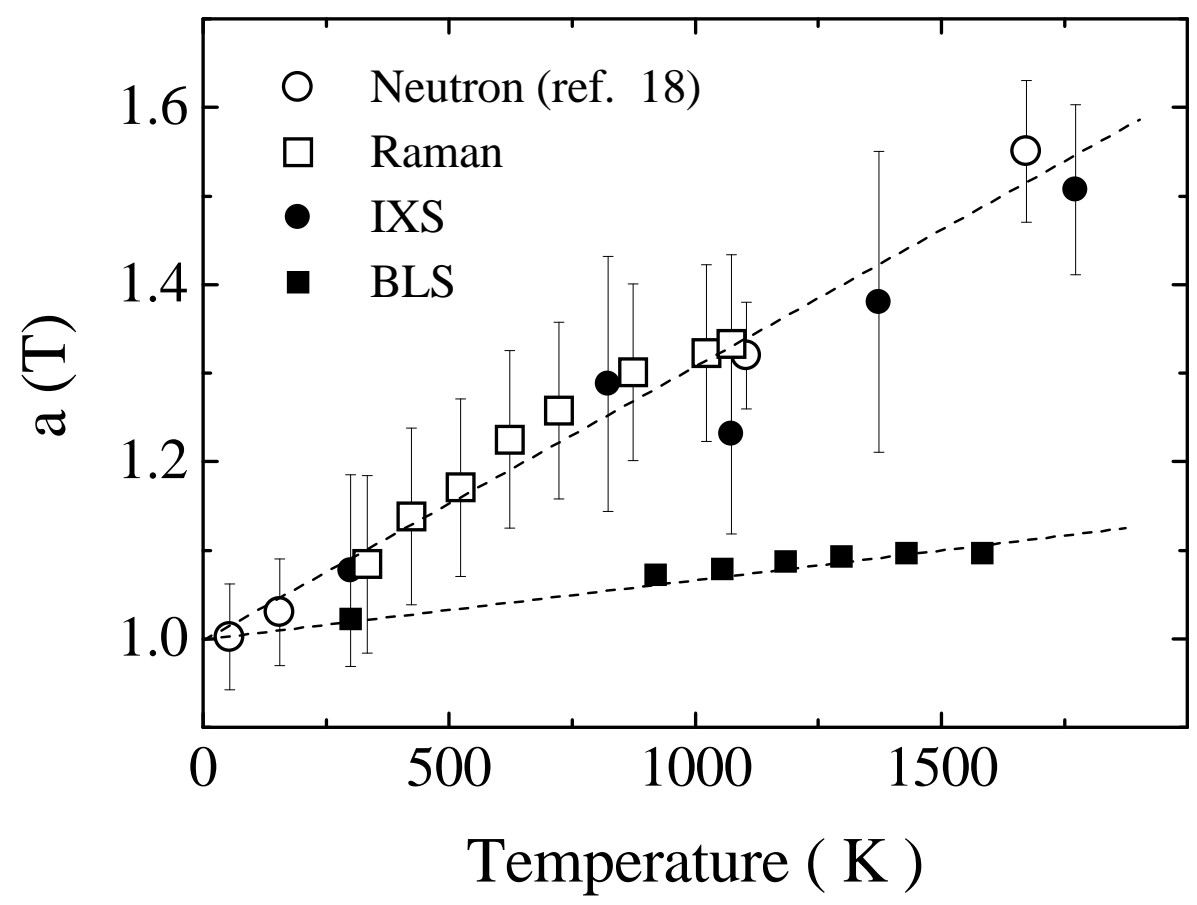

Figure 3: "Acoustic Nature of the Boson Peak...", by C. Masciovecchio et al. 


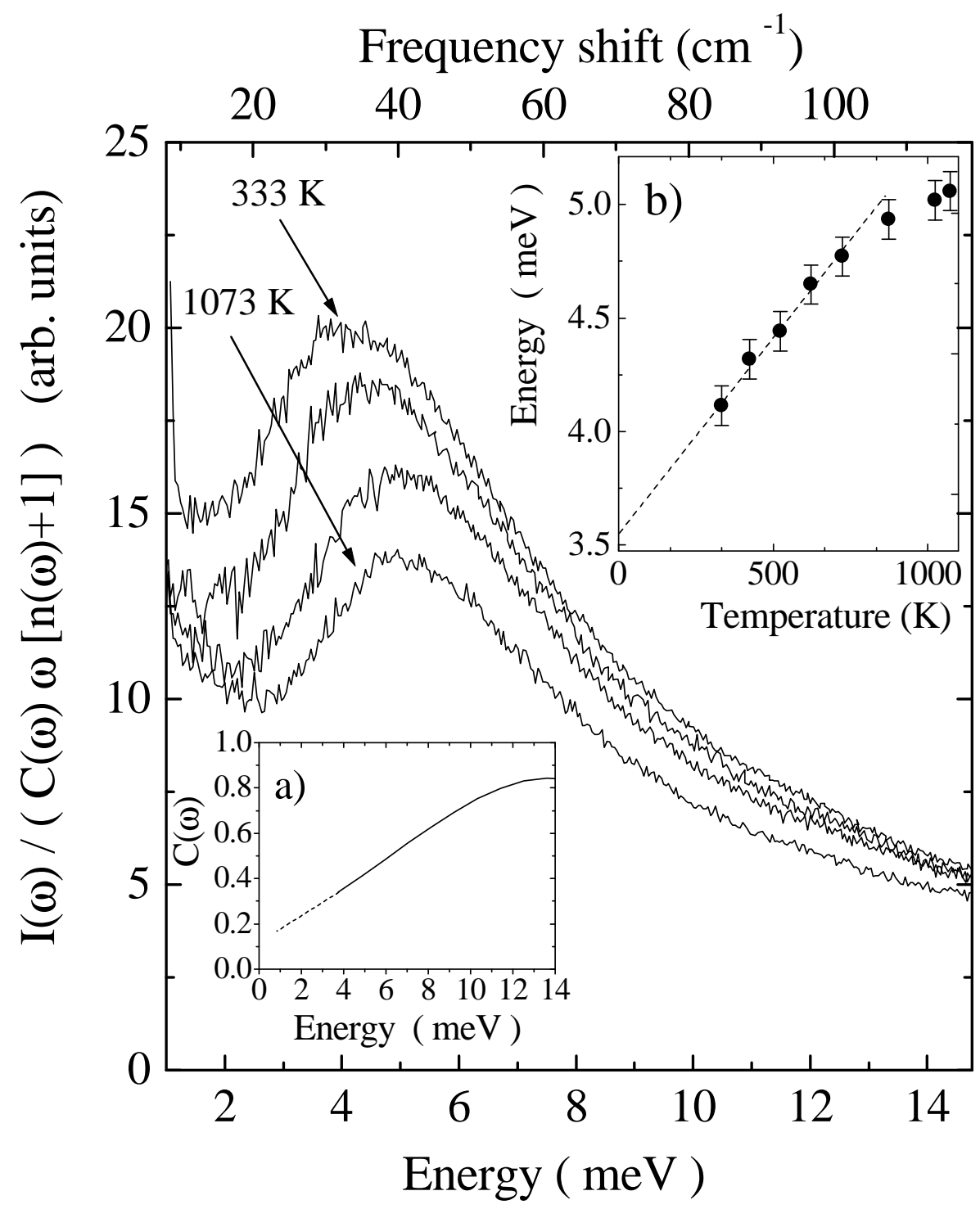

Figure 4: "Acoustic Nature of the Boson Peak...", by C. Masciovecchio et al. 